\title{
QUALIDADE DE FRUTOS DE ACESSOS DE CAJÁ-MANGUEIRA DURANTE A MATURAÇÃO
}

\author{
JOSÉ ROBERTO CHAVES NETO \\ LUANA FERREIRA DOS SANTOS ${ }^{2}$ \\ RENATO LIMA DANTAS ${ }^{3}$ \\ ANA LIMA DANTAS ${ }^{4}$ \\ ANA PAULA PEREIRA SCHUNEMANN ${ }^{5}$ \\ SILVANDA DE MELO SILVA ${ }^{6}$
}

\begin{abstract}
Este estudo teve como objetivo avaliar as mudanças nas características físicas e físico-químicas ocorridas durante a maturação de frutos de acessos de cajá-mangueira (SpondiasdulcisParkinson). Os frutos foram oriundos de cinco plantas de cajá-mangueira, de ocorrência espontânea, em cinco estádios de maturação (TV: totalmente verde; IP: início de pigmentação amarela; $A E$ : amarelo esverdeado e PA: predominantemente amarelo), e posteriormente selecionados quanto à uniformidade da maturação. $O$ delineamento experimental utilizado foi 0 inteiramente casualizado, em esquema bifatorial5 $\times 4$, sendo cinco acessos, quatro estádios de maturação. Os frutos foram avaliados quanto a Acidez Titulavel (AT), teor de sólidos solúveis (SS) e relação SS/AT. Constatou-se que com o avanço da maturação os frutos de todos os acessos apresentam um aumento no diâmetro e comprimento, na massa fresca, e um aumento na luminosidade e na intensidade da coloração da casca, em paralelo a transição da cor verde para uma cor em tons de amarelo intenso. De modo geral os frutos da cajá-mangueira apresentam rendimento de polpa superior a $70 \%$, sendo esta porcentagem superior ao mínimo aceito para industrialização. Os frutos dos acessos P1, P2 e P5 destacam-se dupla finalidade, tanto para o consumo fresco como para a industrialização, por apresentarem rendimento de polpa, teor de SS superior e AT, superior ao mínimo aceitável pelo padrão de identidade e qualidade (PIQ). Além de elevado conteúdo de ácido ascórbico, destacando-se os acessos $\mathrm{P} 1$ e $\mathrm{P} 2$ formam superiores aos demais por terem apresentado maior conteúdo de ácido ascórbico.
\end{abstract}

PALAVRAS-CHAVE: SPONDIASDULCISPARKINSON; FRUTÍFERA EXÓTICA; ANÁLISES FÍSICAS;

'Engenheiro Agrônomo, Doutorando em Engenharia Agrícola - Universidade Federal de Santa Maria (UFSM), Santa Maria, RS, Brasil. (e-mail: jose.chavesneto@gmail.com). \# Autor para correspondência.

${ }^{2}$ Engenheira Agrônoma, Doutoranda em Agronomia - Universidade Federal da Paraíba (UFPB), Areia, PB, Brasil. (e-mail: sferreira.luana@gmail.com).

${ }^{3}$ Engenheiro Agrônomo, Doutor em Agronomia, Docente na Faculdade de Enfermagem Nova Esperança (FACENE), João Pessoa, PB, Brasil. (e-mail: renatodsobrinho@gmail.com).

${ }^{4}$ Engenheira Agrônoma, Doutora em Agronomia, Editora Científica da Faculdade de Enfermagem Nova Esperança (FACENE), João Pessoa, PB, Brasil. (e-mail: dantas.ana.lima@gmail.com).

${ }^{5}$ Engenheira Agrônoma, Doutora em Agronomia, Florianópolis, SC, Brasil. (e-mail: anaschunemann@gmail.com).

${ }^{6}$ Professora Adjunta no Departamento de Ciências Fundamentais e Sociais, Universidade Federal da Paraíba (UFPB), Areia, PB, Brasil. (e-mail: silvandasilva@gmail.com). 


\section{INTRODUÇÃO}

O gênero Spondias, pertencente à família Anacardiace, é composto por 18 espécies distribuídas nos neotrópicos, Ásia e Oceania. No Brasil algumas dessas espécies ocorrem de forma espontânea ou subespontânea do litoral até o Semiárido brasileiro, grande parte destas espécies são encontradas na região nordeste do Brasil, que é rica em espécies frutíferas nativas e exóticas não tradicionais, dentre as espécies pertencente ao gênero Spondias, destacam-se algumas como: Spondiasmombin L. (cajazeira), Spondiaspurpurea L. (cirigueleira), SpondiascythereaSonn. (cajaraneira ou cajá-mangueira), Spondias tuberosa Arr. Câm. (umbuzeiro) e Spondiassp. (umbu-cajazeira e umbugueleira), estas são consideradas frutas "potenciais" devido as suas excelentes características organolépticas, bem como pelo elevado consumo e aceitação pela população das áreas de ocorrência durante as safras (Lorenziet al., 2006; Silva et al., 2012; Moura et al., 2013).

A cajá mangueira (SpondiasdulcisParkinson) é uma espécie frutífera exótica no Brasil, que temsua origem na llha do Pacífico, e está presente em quase todo território brasileiro, sendo encontrada distribuída na zona da mata, no agreste, nas regiões semi-áridas, sub-úmida e semi-úmida do nordeste brasileiro. Seus frutos são elipsoides, classificados como drupa, que possuem um caroço dotado de fibras rígidas e espinescentes que mergulham parcialmente na polpa. Sua polpa é rica em fibras, sais e algumas vitaminas ( $A, B 1, B 6$ e $C$ ) e grande potencial agroindustrial, sendo matéria prima para fabricação de diversos produtos (sucos, coquetéis, batidas, licores e sorvetes), apresentando relevante aspecto comercial em função de seu aroma, sabor e palatabilidade e potencial na agroindústria. No entanto, o extrativismo é sua principal forma de exploração, sendo destinados principalmente ao consumo de fruta fresca (Lorenziet al., 2006; Moura et al., 2013).

São conhecidos dois tipos de cajá-mangueira, a variedade grande com frutos de 8-10 $\mathrm{cm}$ de comprimento, diâmetro 5-6 cm, e um peso médio de 120-200 g, tendo a árvores um porte médio variando de $10-20 \mathrm{~m}$ de altura, enquanto a variedade anã apresenta frutos medindo de 50-60 mm cm de comprimento, diâmetro de 40-50 mm e um peso médio de 50-70 g (Grahamet al., 2004; Franquin et al., 2008). Seus frutos são elipsoides, classificados como drupa de formato cilíndrico, com massa média variando de 100a $380 \mathrm{~g}$ e a semente apresenta feixes duros e longos profundamente internados na polpa dos frutos (Gomes, 2007).

Os frutos de cajá-mangueira quando maduros pesam em média 136,3 g, com um diâmetro longitudinal em média de $76,68 \mathrm{~mm}$ e transversal de $54,73 \mathrm{~mm}$ e um rendimento de polpa em média de $61,02 \%$ (Damiani et al., 2011). Sua polpa quando madura torna-se amarelo pálida, sumarenta, acídula e doce, que cobre uma semente eriçada de compridos feixes lenhosos, entranhados profundamente na massa da polpa. Estes frutos são ricos em fibras, sais e algumas vitaminas (A, B1, B6 e C), com teores de sólidos solúveis (SS) superiores aos de outras Spondias, segundo Kohatsuet al. (2011), o fruto da cajá-mangueira apresenta valores médios de 14,23 e 15,33\%, na pós-colheita, enquanto que frutos de umbuzeiro, de acordo com Dantas Júnior (2008), apresentam valor máximo foi de $12,5 \%$ de SS.

No processo de maturação, os frutos passam por uma série de modificações nas características externas (aparência) e internas (composição química), desdea maturidade fisiológica, passando pela pré-maturação, maturaçãoe por fim atingindo o amadurecimento, ponto em que os frutosatingem sua máxima qualidade (Chitarra\&Chitarra, 2005). As principais modificações nas características de qualidadesão observadas na cor da casca,taxa respiratória, produção de etileno, firmeza dos frutose rendimento de polpa, além de aumentona 
doçura, em paralelo a elevação do teor de sólidos solúveis e decréscimo na acidez, aumentando as características do aroma e sabor, devido emanação de compostos voláteis (Silva, 2010; Silvaet al., 2013). Tais características possuem grande influência na aceitação de determinado fruto não só para indústria, mas também para o consumo do fruto fresco pelos consumidores.

Está espécie apresenta grande potencial para comercialização na região Nordeste. Tendo em vista a falta de informações no Brasil sobre as características físicas e físicoquímicas de frutos de cajá-mangueira e a influência que estas empregam na aceitação destes frutos para o consumo in natura e para indústria, este trabalho teve por finalidade avaliar as mudanças nos aspectos físicos e físico-químicos de qualidade ocorridas durante a maturação de frutos de diferentes acessos de cajá-mangueira (SpondiasdulcisParkinson).

\section{MATERIAL E MÉTODOS}

O trabalho foi conduzido entre os meses de janeiro e maio de 2013, no Laboratório de Biologia e Tecnologia de Pós Colheita do Departamento de Ciências Fundamentais e Sociais do Centro de Ciências Agrárias, Campus II da Universidade Federal da Paraíba (DCFS/CCA/UFPB), Areia/Paraíba. Os frutos foram colhidos no período da manhã de cinco acessos de cajá-mangueira (P1, P2, P3, P4, P5) de ocorrência espontânea no Estado da Paraíba, e transportados ao laboratório.

No laboratório os frutos foram lavados e selecionados quanto à presença de danos físicos ocasionados pelo manuseio e transporte, pelo ataque de doença ou praga bem como pelo estádio de maturação, de acordo com o grau de coloração da casca, sendo classificados em quatro estádios de maturação (TV - Totalmente Verde; IP - Início de Pigmentação Amarela; AE - Amarelo Esverdeado e PA - Predominantemente Amarelo) (Figura 1).

\section{FIGURA 1 - EVOLUÇÃO DA MATURAÇÃO DE FRUTOS DE ACESSOS DE CAJÁ- MANGUEIRA (Spondiasdulcis Parkinson) DE OCORRÊNCIA NO ESTADO DA PARAÍBA}

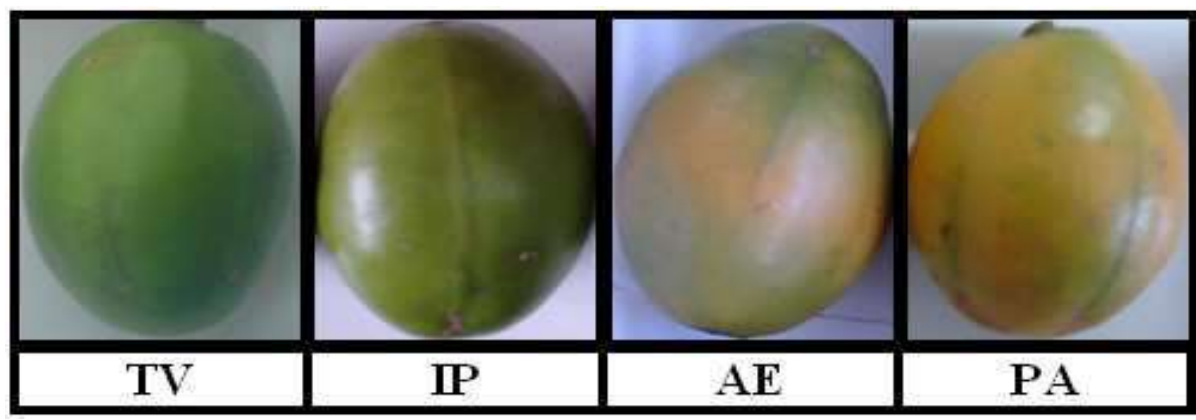

\subsection{CARACTERÍSTICAS FÍSICAS}

Para as avaliações físicas foram utilizados 15 frutos de cada estádio de maturação e de cada acesso, sendo cada fruto considerado uma repetição. Comprimento e Diâmetro $(\mathrm{mm})$, determinado por medições realizadas com o auxílio do paquímetro digital; massa fresca do fruto $(\mathrm{g})$, determinada através de pesagem direta, utilizando-se balança semi-analítica, A $42207 \mathrm{c}$ - Bel Engeneering e rendimento de polpa (\%), determinada através do isolamento e 
pesagem da parte em balança semi-analítica, A 42207c - Bel Engeneering. A coloração da casca, foi realizada através de avaliação objetiva, com o auxílio de um colorímetro digital Minolta (CR-300), o qual expressa à cor nos parâmetros: $L^{*}$ (corresponde à claridade/luminosidade); $\mathrm{a}^{*}$ (define a transição da cor verde $\left(-\mathrm{a}^{*}\right)$ para a cor vermelha $\left.\left(+\mathrm{a}^{*}\right)\right)$ e $b^{*}$ (representa a transição da cor azul $\left(-b^{*}\right)$ para a cor amarela $\left(+b^{*}\right)$, onde quanto mais distante do centro $(=0)$, mais saturada a cor).

\subsection{CARACTERÍSTICAS FÍSICO-QUÍMICA}

Para as avaliações físico-químicas foram utilizadas três repetições, realizadas em triplicata, a partir do processamento de cerca de $300 \mathrm{~g}$ de polpa/repetição. Acidez Titulavel (AT - \% de Ácido Cítrico), determinada por titulometria com Hidróxido de Sódio 0,1M (Gondimet al., 2013); teor de sólidos solúveis (SS), determinado com refratômetro digital de marca ATAGO PR-101, com escala variando de 0 a 45 Brix e expresso em \% (AOAC, 2005) e a relação SS/AT (Sólidos Solúveis/ Acidez Titulável), obtida pela divisão simples dos valores de SS pelos de AT. O conteúdo de ácido ascórbico, foideterminado por titulometria utilizando-se solução de DFI (2,6-dicloro-fenol-indofenol 0,002\%) até obtenção de coloração roseo claro permanente, utilizando-se cerca de $1 \mathrm{~g}$ da amostra em $50 \mathrm{ml}$ de Ácido Oxálico 0,5\% conforme (Strohecker\&Henning, 1967).

\subsection{DELINEAMENTO EXPERIMENTAL E ANÁLISE ESTATÍSTICA}

O experimento foi conduzido em delineamento inteiramente casualizado, em esquema bifatorial5 $\times 4$, sendo cinco plantas e quatro estádios de maturação. Os resultados foram submetidos a análise de variância (ANOVA), e as médias comparadas pelo teste de Tukey a $5 \%$ de probabilidade de erro $(p \leq 0,05)$, através do programa estatístico SISVAR versão 5.6 (Ferreira, 2014).

\section{RESULTADOS E DISCUSSÃO}

Quanto ao comprimento $(\mathrm{mm})$ dos frutos de cajá-mangueira houve diferença significativa $(p \leq 0,05)$, tanto entre os estádios de maturação em relação aos acessos, como também entre os acessos em relação aos estádios de maturação estudados (Tabela 1).

De modo geral para todos os acessos, o maior incremento de comprimento ocorreu do estádio TV para o IP, com valores de 60,18 e 64,24mm, respectivamente (Tabela 1). Os acessos P1, P2 e P5, no entanto, apresentaram declínio no comprimento no estádio AE, e o comprimento dos acessos $\mathrm{P} 1$ e P2 não diferiram a partir do primeiro estádio (TV).

No estádio TV, o comprimento dos frutos dos acessos P2, P5 e P1 foi superior significativamente, com 64,07, 64,04 e 62,21 mm. No estádio IP, o P5 foi superior estatisticamente por apresentar frutos com maior comprimento, com média de $74,60 \mathrm{~mm}$, e os menores para os $\mathrm{P} 4$ e P3, com 56,51 e 57,96 mm, respectivamente (Tabela 1).

No estádio de maturação $\mathrm{AE}$, o acesso P5 apresentou maior comprimento, com média de 72,06 mm, sendo os menores de P3 e P4, com 60,09 e 58,04 mm, respectivamente. No estádio PA, apenas o comprimento do P5 foi superior aos demais, com $82,73 \mathrm{~mm}$, e os menores foi de P1, P2, P3 e P4, com média variando entre 63,70 a 58,49 mm (Tabela 1).

Os valores de comprimento deste trabalho são superiores aos reportados para 0 mesmo fruto por Silva et al. (2009), com comprimento de 39,10 $\mathrm{mm}$ para frutos do estádio 
verde e de 41,70 mm para estádio maduro. Porém, são inferiores aos reportados por Damiani et al. (2011), com comprimento médio de $76,68 \mathrm{~mm}$ para esse fruto avaliado no estádio maduro, correspondente ao estádio TA deste trabalho.

TABELA 1 - COMPRIMENTO (mm) DE FRUTOS DE DIFERENTES ACESSOS DE CAJÁMANGUEIRA EM DIFERENTES ESTÁDIOS DE MATURAÇÃO

\begin{tabular}{|c|c|c|c|c|c|c|}
\hline \multirow{2}{*}{ Acessos } & \multicolumn{4}{|c|}{ Estádios de Maturação } & \multirow{2}{*}{$\begin{array}{l}\text { Média } \\
\text { Geral }\end{array}$} & \multirow{2}{*}{ C.V. (\%) } \\
\hline & TV & IP & $\mathrm{AE}$ & PA & & \\
\hline P1 & 62,21Aab & $65,26 \mathrm{Ab}$ & $63,87 \mathrm{Abc}$ & $63,70 \mathrm{Ab}$ & 63,75 & 7,83 \\
\hline $\mathrm{P} 2$ & $64,07 \mathrm{Aa}$ & $66,89 A b$ & $66,04 \mathrm{Ab}$ & $62,95 \mathrm{Ab}$ & 65,06 & 9,47 \\
\hline P3 & $57,88 \mathrm{Bb}$ & $57,96 \mathrm{Bc}$ & $60,09 \mathrm{ABcd}$ & $61,36 \mathrm{Ab}$ & 59,31 & 5,46 \\
\hline P4 & $52,71 \mathrm{Bc}$ & $56,51 \mathrm{Ac}$ & $58,04 \mathrm{Ad}$ & $58,49 A b$ & 56,44 & 6,50 \\
\hline P5 & $64,04 \mathrm{Ca}$ & $74,60 \mathrm{Ba}$ & $72,06 \mathrm{Ba}$ & $82,73 \mathrm{Aa}$ & 71,70 & 6,32 \\
\hline Média Geral & 60,21 & 64,24 & 64,03 & 63,59 & - & - \\
\hline C.V. (\%) & 7,25 & 6,85 & 7,41 & 7,93 & - & - \\
\hline
\end{tabular}

Assim como para o comprimento, o diâmetro diferiu significativamente $(p \leq 0,05)$ com o avanço da maturação para os acessos P3, P4 e P5 (Tabela 2), com maiores valores para frutos do estádio PA, com 45,48, 49,84 e $68,85 \mathrm{~mm}$, e os menores para o estádio mais imaturo TV, com 42,28, 43,69 e 55,67 mm para os acessos P3, P4 e P5, respectivamente.

O estádio de maturação TV do acesso P5 apresentou os frutos com maior diâmetro, com 55,67 mm, sendo os menores os dos acessos P3 e P4, com de 42,28 e 43,69 mm, respectivamente. Para o estádio IP, o acesso P5 apresentou os frutos com maior diâmetro, com 63,97 mm e o menor para o P3 (43,30 mm) (Tabela 2).

O acesso P5 apresentou maior diâmetro no estádio de maturação $\mathrm{AE}$, com 61,13 mm e o menor em frutos do acesso P3 $(45,98 \mathrm{~mm})$. No estádio PA, frutos do acesso P5 apresentaram maior diâmetro, com $68,85 \mathrm{~mm}$, e os acessos P2 e P3 apresentaram os menores, com 47,60 e 45,48 mm, respectivamente (Tabela 2).

Os valores de diâmetro dos frutos neste trabalho são superiores aos de Silva et al. (2009), com diâmetro de 35,02 mm para o estádio de maturação verde e de 43,90 mm para estádio maduro. Porém, próximos aos de Franquinet al. (2008), que descreveram diâmetros de frutos variando entre $50-60 \mathrm{~mm}$.

\section{TABELA 2 - DIÂMETRO (mm) DE FRUTOS DE DIFERENTES ACESSOSDA CAJÁ- MANGUEIRAEM DIFERENTES ESTÁDIOS DE MATURAÇÃO}

\begin{tabular}{|c|c|c|c|c|c|c|}
\hline \multirow{2}{*}{ Acessos } & \multicolumn{4}{|c|}{ Estádios de Maturação } & \multirow{2}{*}{$\begin{array}{l}\text { Média } \\
\text { Geral }\end{array}$} & \multirow{2}{*}{ C.V. (\%) } \\
\hline & TV & $\mathrm{IP}$ & $\mathrm{AE}$ & PA & & \\
\hline P1 & $48,28 \mathrm{Bb}$ & $51,69 A b$ & $50,19 \mathrm{ABbc}$ & $50,61 \mathrm{ABb}$ & 50,16 & 5,42 \\
\hline P2 & $48,33 \mathrm{Ab}$ & $51,48 A b$ & $50,82 A b$ & $47,60 \mathrm{Abc}$ & 49,62 & 9,29 \\
\hline P3 & $42,28 \mathrm{Cc}$ & $43,30 \mathrm{BCd}$ & $45,98 A c$ & $45,48 \mathrm{ABc}$ & 44,27 & 5,91 \\
\hline P4 & $43,69 B c$ & $47,73 \mathrm{Ac}$ & $49,11 \mathrm{Abc}$ & $49,84 \mathrm{Abc}$ & 47,59 & 7,09 \\
\hline P5 & $55,67 \mathrm{Aa}$ & $63,97 \mathrm{Ba}$ & $61,13 \mathrm{Ba}$ & $68,85 \mathrm{Aa}$ & 61,27 & 5,59 \\
\hline Média Geral & 47,72 & 51,63 & 51,56 & 50,39 & - & - \\
\hline C.V. (\%) & 6,06 & 6,17 & 7,83 & 7,23 & - & - \\
\hline
\end{tabular}

O comprimento e diâmetro são as características que dão a ideia do formato do fruto. O aumento no tamanho dos frutos durante a maturação se dá por meio da expansão celular, o 
que resulta em um aumento do comprimento e diâmetro, que se estabilizam no amadurecimento (Chitarra\&Chitarra, 2005). Os frutos de cajá-mangueira apresentam comprimento e diâmetro superiores aos de outras Spondias, Silva (2010) reportou para cajá comprimento variando de 32,00 a 39,66 mm e para o diâmetro uma variação de 26,95 a 28,70 $\mathrm{mm}$. Santos et al. (2010), em frutos da umbu-cajazeira, reportou comprimento de 43,80 mm e diâmetro de $30,10 \mathrm{~mm}$. Portanto, com base no tamanho, característica está que torna o fruto mais apreciado para o consumo fresco, os frutos de cajá-manga são mais atrativos para o consumidor que os de outras Spondias.

A massa fresca do cajá-manga diferiu entre os acessos P3, P4 e P5 (Tabela 3), acumulando-se com o avanço da maturação até o estádio PA, visto que a maior massa dos frutos dos acessos foi evidenciada neste estádio, com $69,48 \mathrm{~g}$ para o $\mathrm{P} 3,78,89 \mathrm{~g}$ para o $\mathrm{P} 4$ e 0 P5 com 141,16 g.

O acesso P5 apresentou a maior massa fresca no estádio TV, com 111, $69 \mathrm{~g}$, e as menores massas foram para o P3 com 56,67 g e 56,69 g para o P4. Para o estádio IP, obtevese $60,54 \mathrm{~g}$ na massa fresca dos frutos do P3 a 124,53 g do P5 (Tabela 3).Para o estádio de maturação AE, e a massa fresca dos frutos de cajá-mangueira variou de $68,09 \mathrm{~g}$ no P3 a $141,16 \mathrm{~g}$ no P5. O acesso P5 destacou-se dos demais pela maior massa fresca dos frutos em todos os estádios avaliados (Tabela 3).

Os resultados de massa fresca de frutos de cajá-mangueira deste trabalho, são inferiores aos de Damiani et al. (2011), que reportaram 136,0 g para frutos maduros desta mesma espécie. Porém, superiores aos de Silva et al. (2009), que constataram frutos de cajámanga com massa variando de 25,31 g no estádio verde para 28,90 g no estádio maduro.

A massa fresca do cajá-manga é maior do que a de outras Spondias, Dantas et al. (2016), reportaram para umbuguela (Spondias sp.) massa fresca média de 10,63 g, no amadurecimento pleno na planta. Para umbu-cajá (Spondiassp.) Gondim et al. (2013), observaram média de 28,82 g, quando totalmente amarelo. Lira Junioret al. (2014), constataram massa fresca de ciriguelas(S.purpurea) variando de 9,66 a 10,43 g. A massa fresca apresenta grande importância para o mercado de frutas frescas, visto que frutos com maiores massas e tamanho são mais atrativos para os consumidores.

\section{TABELA 3 - MASSA FRESCA (g) DE FRUTOS DE DIFERENTES ACESSOSDA CAJÁ- MANGUEIRAEM DIFERENTES ESTÁDIOS DE MATURAÇÃO}

\begin{tabular}{|c|c|c|c|c|c|c|}
\hline \multirow{2}{*}{ Acessos } & \multicolumn{4}{|c|}{ Estádios de Maturação } & \multirow{2}{*}{$\begin{array}{l}\text { Média } \\
\text { Geral }\end{array}$} & \multirow[b]{2}{*}{ C.V. (\%) } \\
\hline & TV & IP & $A E$ & PA & & \\
\hline P1 & $82,78 \mathrm{Ab}$ & $97,19 A b$ & $89,08 \mathrm{Ab}$ & $92,71 \mathrm{Ab}$ & 90,42 & 16,82 \\
\hline P2 & $87,04 \mathrm{Ab}$ & $97,49 A b$ & $91,29 A b$ & $84,58 \mathrm{Abc}$ & 90,29 & 24,59 \\
\hline P3 & $56,67 \mathrm{Bc}$ & $60,54 \mathrm{ABc}$ & $68,09 A c$ & $69,48 A c$ & 63,72 & 15,32 \\
\hline P4 & $56,69 \mathrm{Bc}$ & $73,11 \mathrm{Ac}$ & $74,14 \mathrm{Abc}$ & $78,89 A b c$ & 70,71 & 14,81 \\
\hline P5 & $111,69 \mathrm{Ba}$ & $124,53 \mathrm{ABa}$ & $118,95 \mathrm{Ba}$ & $141,16 \mathrm{Aa}$ & 121,07 & 13,69 \\
\hline Média Geral & 79,27 & 90,57 & 88,24 & 86,82 & - & - \\
\hline C.V. (\%) & 16,63 & 16,96 & 20,90 & 16,57 & - & - \\
\hline
\end{tabular}

Para o rendimento de polpa de frutos de cajá-mangueira houve diferença significativa $(p \leq 0,05)$ entre os estádios de maturação para os acessos P1, P2, P4 e P5 (Tabela 4). No geral, ocorreu um aumento no rendimento do estádio TV para IP, estabilizando nos demais para todos os acessos. Os frutos com maior rendimento de polpa foram os do $\mathrm{P} 2$, enquanto os dos TV e IP foram os maiores, com 82,10 e $82,02 \%$, respectivamente, devido o maior 
comprimento e diâmetro e, consequentemente, frutos maiores e com maiores porcentagens de massa fresca, e menores de casca e semente.

Os acessos diferiram significativamente $(p \leq 0,05)$ quanto ao rendimento de polpa nos estádios de maturação TV, IP e PA. No estádio de maturação TV, os acessos P1 e P2, apresentaram as maiores porcentagens de rendimento de polpa, com 81,02 e 82,10\%, respectivamente. Para o estádio IP, as maiores porcentagens de polpa foram dos frutos dos P1, P2, P3 e P5, variando de 82,08 a 79,56\%, e a menor do P4, com 75,96\%. O rendimento de frutos do estádio $A E$, do acesso $P 5$ foi de $81,40 \%$, a maior do estádio $P A$, e as menores foram as dos P1, P2, P3 e P4, que variaram de 76,71 a 78,08\% (Tabela 4).

O rendimento em polpa de frutos de cajá-manga deste trabalho, são superiores aos citados para frutos desta mesma espécie por Damianiet al. (2011) e Silva et al. (2009) que apresentaram rendimento de polpa de $61,02 \mathrm{e} 73,58 \%$, respectivamente, para frutos maduros (correspondente ao estádio TA) desta Spondias.

O cajá manga apresenta rendimento de polpa próximo ao de outras Spondias, Gondim et al. (2013) e Santos et al. (2010) reportaram em umbu-cajá (Spondias sp.) na maturidade comercial rendimento de polpa de 69,70 e $74 \%$, respectivamente, e em umbu ( $S$. tuberosa), Campos (2007) reportou média de 76\%. Com base nisso, destaca-se então o potencial para industrialização dos frutos de cajá-mangueira estudados neste trabalho, visto que os acessos P1, P2 e P5, apresentaram porcentagens de rendimento superiores aos de outras Spondias, com médias de 79,72, 80,03 e 79,62\%, respectivamente.

De acordo com Pinto (2003), na agroindústria, os frutos que apresentam grande potencial industrial são aqueles provenientes dos acessos com rendimento acima da média e baixa acidez. Os frutos estudados neste trabalho apresentaram rendimento de polpa superior a 70\%, sendo superior ao mínimo aceitável para a industrialização, uma vez que, o valor mínimo, de acordo com o Padrão de Identidade e Qualidade (PIQ) do Ministério da Agricultura, Pecuária e do Abastecimento (MAPA) é de 40\% (BRASIL, 1999). Portanto, este é um atributo de qualidade, importante quando estes frutos apresentam potencial para a elaboração de produtos (Costa et al., 2015).

TABELA 4 - RENDIMENTO DE POLPA (\%) DE FRUTOS DE CAJÁ-MANGUEIRA COLHIDOS DE DIFERENTES ACESSOSEM DIFERENTES ESTÁDIOS DE MATURAÇÃO

\begin{tabular}{|c|c|c|c|c|c|c|}
\hline \multirow[b]{2}{*}{ Acessos } & \multicolumn{4}{|c|}{ Estádios de Maturação } & \multirow{2}{*}{$\begin{array}{l}\text { Média } \\
\text { Geral }\end{array}$} & \multirow[b]{2}{*}{ C.V. (\%) } \\
\hline & TV & IP & $\mathrm{AE}$ & $\overline{P A}$ & & \\
\hline $\mathrm{P} 1$ & $81,07 \mathrm{Aa}$ & $81,69 \mathrm{Aa}$ & $77,85 \mathrm{Ba}$ & $76,71 \mathrm{Bb}$ & 79,72 & 3,28 \\
\hline P2 & $82,10 \mathrm{Aa}$ & $82,08 \mathrm{Aa}$ & $78,68 \mathrm{ABa}$ & $76,81 \mathrm{Bb}$ & 80,03 & 4,82 \\
\hline P3 & $78,09 A b$ & $79,92 \mathrm{Aa}$ & $77,94 \mathrm{Aa}$ & $78,08 \mathrm{Ab}$ & 78,52 & 8,44 \\
\hline P4 & $75,08 \mathrm{ABc}$ & $75,96 \mathrm{ABb}$ & $73,71 \mathrm{Ba}$ & $77,93 \mathrm{Ab}$ & 75,67 & 4,27 \\
\hline P5 & $80,60 \mathrm{ABab}$ & $79,56 \mathrm{ABa}$ & $77,98 \mathrm{Ba}$ & $81,40 \mathrm{Aa}$ & 79,62 & 3,05 \\
\hline Média Geral & 79,41 & 79,84 & 77,18 & 77,85 & - & - \\
\hline C.V. (\%) & 3,39 & 4,27 & 8,34 & 3,47 & - & - \\
\hline
\end{tabular}

${ }^{*}$ Médias seguidas de letras iguais, maiúsculas na linha e minúsculas na coluna, não diferem entre si pelo teste de Tukey, ao nível de $5 \%$ de probabilidade.

Durante o crescimento e a maturação dos frutos, ocorre o acúmulo de massa provocando o aumento no volume e nas dimensões dos frutos, o que evidencia a elevada correlação entre as dimensões e o aumento massa fresca ao longo da maturação, tais mudanças são fortemente reguladas por hormônios (Mcateeet al., 2013; Dantas et al., 2016). 
As mudanças na luminosidade $\left(L^{*}\right)$ de frutos da cajá-mangueira são apresentados na Tabela 5. Houve diferença significativa $(p \leq 0,05)$, tanto entre os estádios de maturação em relação aos acessos, com também entre os acessos em relação aos estádios de maturação estudados.

A luminosidade $\left(L^{*}\right)$ dos frutos aumentou como indicado pela elevação dos valores de $L^{*}$ para todos os acessos avaliados. Em média, os valores aumentaram de 38,80 a 45,38 do estádio de maturação TV para o estádio PA. Os frutos de todos os acessos (P1, P2, P3, P4 e P5) apresentaram maior luminosidade $\left(L^{*}\right)$ no estádio de maturação PA (46,37, 45,56, 44,40 e 46,40, respectivamente) (Tabela 5).

Dentre todos os acessos, os que apresentaram maior acréscimo de luminosidade $\left(L^{\star}\right)$ nos frutos durante a maturação, foram os acessos P3 e P5, com valores médios variando do 39,06 a 47,27 do estádio TV para o TA, respectivamente (Tabela 5). Os resultados de luminosidade $\left(L^{*}\right)$ para o cajá-manga corroboram com os reportados por Silva (2010) em cajá que reportaram luminosidade entre 20,00 e 55,00 em frutos de sete estádios de maturação.

Para a característica $a^{*}$ da coloração de frutos da cajá-mangueira, foram observadas diferenças significativas ( $\mathrm{p} \leq 0,05)$, tanto entre os estádios como entre os acessos (Tabela 5 ). O parâmetro de coloração $a^{*}$ indica a mudança da coloração da epiderme do verde (-) para tons que culminam em vermelho (+).

De modo geral, o parâmetro de cor $\mathrm{a}^{*}$ aumentou com o avanço da maturação, passando de 5,22 no estádio de maturação TV para 4,73 no estádio de maturação PA, ou seja, indicando a perda da coloração verdemais escuro, demostrada pelo estádio de maturação TV, mudando o sinal de negativo para positivo no final da maturação, chegando a atingir valores que reportam coloração amarela predominante, demostrada pelo estádio de maturação PA.

O acesso P3 apresentou maior aumento de $a^{*}$ durante a maturação, partindo de $-7,12$ no estádio IP para 13,68 no estádio de maturação TA (Tabela 5). Os valores do parâmetro a* de coloração da casca de cajá-manga apresentaram comportamento similar ao de cajá, conforme relatado por Silva (2010), cujos valores do parâmetro $a^{*}$, com a transição do sinal de negativo $(-10,00)$ para positivo $(15,00)$, aumentaram com a evolução da coloração laranja durante a maturação.

Esta perda da cor verde com o avanço da maturação é tida como uma característica inerente a maioria dos frutos. Dentre as frutíferas do gênero Spondias, esta característica também foi verificada, em frutos de ciriguela (S. purpurea) (Martinset al., 2003), cajá (S.mombin) (Mouraet al., 2003), umbu (S. tuberosa) (Silvaet al., 2011; Mouraet al., 2013) e em umbugueleira (Spondia sp.) (Dantaset al., 2016), posteriormente está cor verde é substituída por outros pigmentos.

De acordo Chitarra e Chitarra (2005), os frutos devido aos pigmentos predominantes e sua distribuição, apresentam uma cor de fundo, que corresponde fundamentalmente às clorofilas e carotenoides, nos tons verde, amarelo e alaranjado.O teor de carotenoides dos frutos aumenta durante a maturação e amadurecimento, sendo que a intensificação da cor é definida pela degradação da clorofila(Silvaet al.,2012).

Os valores do parâmetro $b^{*}$ aumentaram com a maturação nos frutos dos acessos avaliados. Esse aumento, em média, partiu de 36,33 no estádio IP para 61,68 no estádio TA, indicando o surgimento da coloração amarela nos frutos. No estádio de maturação TV, os frutos apresentavam-se com coloração totalmente verde, com valores de $b^{*}$, variando de 32,60 a 49,40, associado aos valores mais negativos de $a^{*}$. O início da coloração amarela foi observado no estádio IP, onde o parâmetro b* variou de 34,52 a 53,82 (Tabela 5). De acordo com Wills e Widjanarko(1995), a mudança da cor verde para amarelo devese à degradação da clorofila e a síntese e revelação de carotenoides e/ou flavonoides amarelos.

Os resultados encontrados neste trabalho, corroboram com Moura et al. (2013), que também observou a perda coloração verde dos frutos de umbu ao longo da maturação, e aumento da intensidade 
da coloração amarela pelas características *a e *b, respectivamente. Martins et al. (2003) e Dantas et al. (2016), observaram durante a maturação de frutos de ciriguela(S. purpurea), que a coloração dos frutos evoluiu do verde para o amarelo, adquirindo, no final tons avermelhados.

Em cajá(S. mombin), Silva (2010) reportou um acréscimo do parâmetro b* na casca dos frutos durante a maturação, sendo este acréscimo evidenciado no início da pigmentação amarela, conforme também observado neste trabalho.

TABELA 5 - MUDANÇAS NOS PARÂMETROS DE COLORAÇÃO L*, a* e b* DA CASCA DE FRUTOS DE DIFERENTES ACESSOSDA CAJÁ-MANGUEIRA EM DIFERENTES ESTÁDIOS DE MATURAÇÃO

\begin{tabular}{|c|c|c|c|c|c|c|}
\hline \multirow{2}{*}{ Acessos } & \multicolumn{4}{|c|}{ Estádios de Maturação } & \multirow{2}{*}{$\begin{array}{l}\text { Média } \\
\text { Geral }\end{array}$} & \multirow[t]{2}{*}{ C.V. (\%) } \\
\hline & TV & IP & $\mathrm{AE}$ & PA & & \\
\hline \multicolumn{7}{|c|}{ Parâmetros de coloração L* } \\
\hline $\mathrm{P} 1$ & $38,29 \mathrm{Cb}$ & $40,70 \mathrm{Bab}$ & $42,15 \mathrm{Ba}$ & $46,37 \mathrm{Aa}$ & 41,49 & 4,37 \\
\hline P2 & $38,01 \mathrm{Bb}$ & $39,89 \mathrm{Bbc}$ & $42,68 \mathrm{Ba}$ & $45,00 \mathrm{Aa}$ & 41,27 & 5,86 \\
\hline P3 & $39,06 \mathrm{Cab}$ & $38,81 \mathrm{Cc}$ & $42,64 \mathrm{Ba}$ & $45,56 \mathrm{Aa}$ & 41,49 & 2,95 \\
\hline P4 & $38,68 \mathrm{Cab}$ & $41,19 \mathrm{Bab}$ & $42,34 \mathrm{Ba}$ & $44,40 \mathrm{Aa}$ & 41,65 & 3,00 \\
\hline P5 & $39,98 \mathrm{Ca}$ & 42,09Ba & $43,77 \mathrm{Ba}$ & $46,40 \mathrm{Aa}$ & 42,47 & 4,12 \\
\hline Média Geral & 38,80 & 40,54 & 42,76 & 45,38 & - & - \\
\hline C.V. (\%) & 3,47 & 4,34 & 4,70 & 4,01 & - & - \\
\hline \multicolumn{7}{|c|}{ Parâmetro de coloração a* } \\
\hline P1 & $-2,46 \mathrm{Ca}$ & $-2,18 \mathrm{Ca}$ & 2,71Ba & $10,87 \mathrm{Aa}$ & 1,49 & 8,15 \\
\hline P2 & $-2,28 \mathrm{Ca}$ & $-1,49 \mathrm{Ca}$ & $5,14 \mathrm{Ba}$ & $8,06 \mathrm{Ab}$ & 2,16 & 9,28 \\
\hline P3 & $-7,12 \mathrm{Cbc}$ & $-5,92 \mathrm{Cb}$ & $-3,02 \mathrm{Bb}$ & $4,31 \mathrm{Ac}$ & $-2,99$ & 6,86 \\
\hline P4 & $-6,08 \mathrm{Cb}$ & $-5,58 \mathrm{BCb}$ & $-5,03 \mathrm{Bbc}$ & $-2,03 A d$ & $-4,68$ & 3,91 \\
\hline P5 & $-8,17 \mathrm{Bc}$ & $-6,77 \mathrm{Bb}$ & $-6,49 B c$ & $4,16 \mathrm{Ac}$ & $-5,81$ & 16,68 \\
\hline Média Geral & $-5,20$ & $-4,39$ & $-1,69$ & 4,73 & - & - \\
\hline C.V. (\%) & 7,77 & 6,17 & 13,20 & 7,76 & - & - \\
\hline \multicolumn{7}{|c|}{ Parâmetro de coloração b* } \\
\hline $\mathrm{P} 1$ & $32,60 \mathrm{Cb}$ & $36,98 \mathrm{Bb}$ & $39,11 \mathrm{Bb}$ & $46,33 A c$ & 38,11 & 6,31 \\
\hline P2 & $32,60 \mathrm{Bb}$ & $35,08 \mathrm{Bb}$ & $39,44 \mathrm{Ab}$ & $42,42 A c$ & 37,21 & 9,56 \\
\hline P3 & $33,93 \mathrm{Cb}$ & $34,52 \mathrm{Cb}$ & $39,53 \mathrm{Bb}$ & $68,75 \mathrm{Aa}$ & 43,94 & 6,97 \\
\hline P4 & $33,09 \mathrm{Db}$ & $35,71 \mathrm{Cb}$ & $37,33 \mathrm{Bb}$ & $40,49 A c$ & 36,65 & 4,22 \\
\hline P5 & $49,40 \mathrm{Ba}$ & $53,82 \mathrm{ABa}$ & $59,31 \mathrm{Aa}$ & $60,06 \mathrm{Ab}$ & 54,87 & 14,86 \\
\hline Média Geral & 36,36 & 39,22 & 43,28 & 50,70 & - & - \\
\hline C.V. (\%) & 13,21 & 7,43 & 10,31 & 9,49 & - & - \\
\hline
\end{tabular}

Os sólidos solúveis (SS) diferiram significativamente $(p \leq 0,05)$ tanto entre os estádios de maturação para todos os acessos, como também entre os acessos para todos os estádios de maturação avaliados (Tabela 6). De modo geral aumentando com o avanço da maturação, iniciando com $8,4 \%$ em frutos no estádio TV para 12,99\% no PA.

Este comportamento de aumento nos SS com o avanço da maturação também foi descrito por Dantas et al. (2016), que reportaram para frutos de umbugueleira (Spondia sp.), um incremento de 10,82 \% estádios iniciais para 20,86 \% ao final da maturação, Campos (2007), observaram para frutos de umbu (S.tuberosa) o maior conteúdo de SS foi de $12,3 \%$ no fruto maduro.No estádio TV, os frutos dos acessos P1, P2 e P3, se destacaram-se com os maiores teores de SS, entre 8,80 e $9,70 \%$. Para o IP, os maiores teores foram os de frutos do P1 e P2, com 10,23 e 9,92\%, respectivamente (Tabela 6).

Os frutos dos acessos P2 e P1 apresentaram os maiores teores de SS no estádio AE, com 13,68 e 12,57\%, respectivamente. No estádio PA, os frutos do acesso P3 destacaram-se por apresentar o maior teor de SS, não diferindo estatisticamente dos acessos P1 e P2, com 
teores variando de 14,40 a $14,95 \%$, e o menor teor constatado para os frutos do acesso P4, com média de 7,92\% (Tabela 6).

Os valores de SS de frutos de cajá-mangueira deste trabalho, corroboram com os de Kohatsuet al. (2011), com 14,23 e 15,33\% para frutos armazenados por 6 dias a $4^{\circ}$ e $25^{\circ} \mathrm{C}$, respectivamente, e também próximos aos citados por Aroucha et al. (2012), que variaram de 8,5 a $11,77 \%$ em quatro estádios de maturação. No entanto, estando abaixo dos citados por Damiani et al. (2011), que reportaram 19,00\% em frutos colhidos maduros.

Com base nos dados obtidos neste trabalho, o teor de sólidos solúveis (SS) de frutos de cajá-mangueira é superior aos encontrados em outras Spondias,no final da maturação. Moura et al. (2013) observaram para frutos de umbu maduro sólidos solúveis de 12,30\%. Silva et al. (2011) e Gondim et al. (2013), reportaram para frutos umbu-cajá (Spondia sp.) maduros teores máximos de sólidos solúveis de 12,46, 12,90\%, respectivamente. No entanto, inferior aos citados por Silva et al. (2013), para frutos de cajá (S.mombin), com conteúdo máximo de SS de $17,43 \%$.

\section{TABELA 6 - CONTEÚDO DE SÓLIDOS SOLÚVEIS - SS (\%) EM FRUTOS DE DIFERENTES ACESSOSDA CAJÁ-MANGUEIRA EM DIFERENTES ESTÁDIOS DE MATURAÇÃO}

\begin{tabular}{|c|c|c|c|c|c|c|}
\hline \multirow[b]{2}{*}{ Acessos } & \multicolumn{4}{|c|}{ Estádios de Maturacão } & \multirow{2}{*}{$\begin{array}{l}\text { Média } \\
\text { Geral }\end{array}$} & \multirow[b]{2}{*}{ C.V. (\%) } \\
\hline & TV & $\mathrm{IP}$ & $\mathrm{AE}$ & PA & & \\
\hline $\mathrm{P} 1$ & $9,20 \mathrm{Ca}$ & $10,23 \mathrm{Ca}$ & $12,57 \mathrm{Ba}$ & $14,40 \mathrm{Aa}$ & $11,60 a$ & 4,69 \\
\hline P2 & $9,70 \mathrm{Ca}$ & $9,92 \mathrm{Ca}$ & $13,68 \mathrm{Ba}$ & $14,70 \mathrm{Aa}$ & $12,00 \mathrm{a}$ & 1,21 \\
\hline P3 & $8,80 \mathrm{Ba}$ & $7,50 \mathrm{Cb}$ & $9,75 \mathrm{Bb}$ & $14,95 \mathrm{Aa}$ & $10,25 b$ & 4,04 \\
\hline P4 & $7,42 \mathrm{ABb}$ & $6,45 \mathrm{Bc}$ & $7,08 \mathrm{ABd}$ & $7,92 \mathrm{Ac}$ & $7,22 \mathrm{c}$ & 5,34 \\
\hline P5 & $6,92 \mathrm{Cb}$ & $8,00 \mathrm{Bb}$ & $8,42 \mathrm{Bc}$ & $13,00 \mathrm{Ab}$ & $9,08 b$ & 4,49 \\
\hline Média Geral & 8,41 & 8,42 & 10,30 & 12,99 & - & - \\
\hline C.V. (\%) & 4,52 & 3,22 & 4,46 & 3,23 & - & - \\
\hline
\end{tabular}

* Médias seguidas de letras iguais, maiúsculas na linha e minúsculas na coluna, não diferem entre si pelo teste de Tukey, ao nível de $5 \%$ de probabilidade.

O teor de sólidos solúveis é tido com um indicativo da quantidade de açúcares solúveis presente nos frutos, elevados teores de sólidos solúveis são importantes, tanto para o consumo da fruta fresca, quanto para a indústria, uma vez que, proporcionam melhor sabor e maior rendimento na elaboração dos produtos, reportando a estes frutos dupla finalidade. (Sacramentoet al., 2007; Silva, 2010), De acordo com Chitarra e Chitarra (2005), durante a maturação o teor de sólidos solúveis tende a aumentar devido a biossíntese de açúcares solúveis ou a degradação de polissacarídeos, como é o caso do amido.

A acidez titulável $(A T)$, diferiu significativamente $(p \leq 0,05)$ tanto entre os estádios de maturação para todos os acessos, como também entre os acessos para todos os estádios de maturação avaliados (Tabela 7).

Constatou que a AT diminuiu com o avanço da maturação de frutos da cajámangueira, de modo que o acesso P3 se destacou com o maior declínio, com valor médio de $1,59 \%$ de ácido cítrico, no estádio TV, para 1,19\% no estádio PA (Tabela 7). De acordo com Chitarra e Chitarra (2005), durante a maturação dos frutos os ácidos orgânicos livres ou combinados a outros compostos, presentes nos vacúolos das células são utilizados como fontes de energia, sendo oxidados no ciclo de Krebs, gerando compostos intermediários importantes para o metabolismo secundário.

Para todos os estádios de maturação avaliados, o acesso P3 apresentou os maiores teores de AT e o P5 os menores. A AT de frutos do P3 variou de 1,59 a 1,19\% e do P5 de 0,79 a $0,69 \%$ de ácido cítrico (Tabela 7 ). 
Lago-Vanzelaet al., (2011), reportaram para cajá-manga predominantemente amarelo 1,75\% de ácido cítrico e Silva et al. (2009) AT de 1,44 a 0,79\% de ácido cítrico no fruto verde e maduro, respectivamente, que também declinou com 0 avanço da maturação. Este comportamento também foi reportado por Arouchaet al. (2012), para frutos de cajá-mangueira, que constataram valor médio de $2,00 \%$ no fruto verde, decaindo para 1,03\% de ácido cítrico no maduro. A redução da acidez titulavel com o avanço da maturação também foi observado em frutos da umbugueleira(Spondiasp.) por Dantas et al. (2016), reduzindo de 1,24 para 0,63 g.100 $\mathrm{g}^{-1}$ de ácido cítrico no amadurecimento pleno na planta.

\section{TABELA 7 - TEOR DE ACIDEZ TITULÁVEL (\% DE ÁCIDO CÍTRICO) DE FRUTOS DE DIFERENTES ACESSOSDA CAJÁ-MANGUEIRA EM DIFERENTES ESTÁDIOS DE MATURAÇÃO}

\begin{tabular}{|c|c|c|c|c|c|c|}
\hline \multirow[b]{2}{*}{ Acessos } & \multicolumn{4}{|c|}{ Estádios de Maturacãa } & \multirow{2}{*}{$\begin{array}{l}\text { Média } \\
\text { Geral }\end{array}$} & \multirow[b]{2}{*}{ C.V. (\%) } \\
\hline & TV & IP & $\mathrm{AE}$ & PA & & \\
\hline P1 & $1,31 \mathrm{Ab}$ & $1,15 \mathrm{ABb}$ & $1,10 \mathrm{Bb}$ & 1,07Bab & $1,16 b$ & 5,54 \\
\hline P2 & $1,31 \mathrm{Ab}$ & $1,20 \mathrm{Bb}$ & $1,06 \mathrm{Cb}$ & $0,95 \mathrm{Db}$ & $1,13 b$ & 2,66 \\
\hline P3 & 1,59Aa & $1,48 \mathrm{ABa}$ & $1,40 \mathrm{Ba}$ & $1,19 \mathrm{Ca}$ & $1,41 \mathrm{a}$ & 2,92 \\
\hline P4 & $1,13 \mathrm{Ac}$ & $1,01 \mathrm{Bc}$ & $0,84 \mathrm{Cc}$ & $0,99 \mathrm{Bb}$ & $0,99 c$ & 2,89 \\
\hline P5 & $0,79 A d$ & $0,80 \mathrm{Ad}$ & $0,68 \mathrm{Bd}$ & $0,69 \mathrm{Bc}$ & $0,74 d$ & 3,99 \\
\hline Média Geral & $1,23 \mathrm{~A}$ & $1,13 \mathrm{~B}$ & $1,01 \mathrm{C}$ & $0,98 \mathrm{C}$ & - & - \\
\hline C.V. (\%) & 3,57 & 3,36 & 3,17 & 4,96 & - & - \\
\hline
\end{tabular}

Segundo Lima et al. (2002), os frutos de maior interesse para a agroindústria, são aqueles que apresentam AT superior a 1,00 \%, o que reduz a necessidade de padronização com a adição de ácido cítrico da polpa, além de inibir o desenvolvimento de microrganismos. Com base nesta faixa AT de interesse, dentre os acessos estudados neste trabalho, destacamse o P1, P2 e P3, com valores superiores a este, com médias de 1,16, 1,13 e 1,41\%, respectivamente (Tabela 7 ).

Segundo Sacramento et al. (2007), a acidez titulável é um dos critérios utilizados para a classificação do sabor do fruto, e um maior teor de acidez eleva a diluição do produto que, por consequência, aumenta o rendimento industrial do suco (Gondimet al., 2013). Essa característica também pode ser utilizada para determinar o ponto de colheita, desde que as diferenças sejam evidenciadas ao longo da maturação (Klugeet al., 2002).

A relação Sólidos Solúveis/AcidezTitulável (SS/AT) aumentou com o avanço da maturação (Tabela 8), em paralelo à redução da AT e ao acúmulo dos SS na polpa dos frutos. A relação SS/AT variou de 7,64 a 14,11 , do estádio TV ao PA. Frutos do acesso P5 apresentaram a maior relação SS/AT em todos os estádios de maturação avaliados, com 11,67 (TV), 12,79 (IP), 18,63 (AE) e 20,93 (PA), devido ao acentuado acúmulo dos SS e redução da AT inferior durante a maturação (Tabela 8).

O aumento da relação SS/AT em paralelo ao amadurecimento também foi observado por Lopes (2007) e Moura et al. (2013), para frutos de frutos de umbu. Os valores da relação SS/AT em frutos de cajá-mangueira constatados neste trabalho, estão próximos aos citados por Silva et al. (2009), que variou de 7,62 a 15,14 do estádio verde para o maduro, respectivamente. Em cajarana, Arouchaet al. (2011) descreveram relação SS/AT variando de 4,2 a 13,2 do estádio de maturação verde para o maduro.

A relação SS/AT do cajá-manga é superior à de outras Spondias no final da maturação, como por exemplo, à do umbu com média de 12,00 (Mouraet al., 2013), à do umbu- 
cajá com valores médios de 7,57, 12,14 e 11,43, Santos et al. (2010), Silva et al. (2011) e Gondim et al. (2013), respectivamente, e para o cajá relação média de 8,64 (Silvaet al., 2013).

De acordo com Dantas et al. (2016), a elevada da relação SS/AT da polpa de frutos indica que estes frutos são muito saborosos para consumo fresco. A relação SS/AT é utilizada como um parâmetro de avaliação do sabor, sendo desejado valores elevados para esta relação, indicando maior conteúdo de açucares em relação aos ácidos (Chitarra\&Chitarra, 2005).

\section{TABELA 8 - RELAÇÃO SÓLIDOS SOLÚVEIS/ACIDEZ TITULÁVEL (SS/AT) DE FRUTOS DE DIFERENTES ACESSOSDA CAJÁ-MANGUEIRA EM DIFERENTES ESTÁDIOS DE MATURAÇÃO}

\begin{tabular}{|c|c|c|c|c|c|c|}
\hline \multirow{2}{*}{ Acessos } & \multicolumn{4}{|c|}{ Estádios de Maturação } & \multirow{2}{*}{$\begin{array}{l}\text { Média } \\
\text { Geral }\end{array}$} & \multirow{2}{*}{ C.V. (\%) } \\
\hline & TV & $\mathrm{IP}$ & $\mathrm{AE}$ & PA & & \\
\hline P1 & $7,00 \mathrm{Dbc}$ & $8,88 \mathrm{Cb}$ & $11,43 \mathrm{Bb}$ & $13,49 A c$ & $10,20 b$ & 4,79 \\
\hline P2 & 7,41Db & $8,24 \mathrm{Cb}$ & $12,96 \mathrm{Bb}$ & $15,55 \mathrm{Ab}$ & $11,04 b$ & 2,26 \\
\hline P3 & $5,55 \mathrm{Cd}$ & $5,09 \mathrm{Cd}$ & $6,99 B \mathrm{Bc}$ & $12,59 A c$ & $7,55 \mathrm{c}$ & 4,89 \\
\hline P4 & $6,55 \mathrm{Bc}$ & $6,40 \mathrm{Bc}$ & $8,40 A c$ & $8,01 \mathrm{Ad}$ & $7,34 \mathrm{c}$ & 4,39 \\
\hline P5 & $11,67 \mathrm{Ba}$ & $12,79 \mathrm{Ba}$ & $18,63 \mathrm{Aa}$ & $20,93 \mathrm{Aa}$ & $16,01 \mathrm{a}$ & 5,81 \\
\hline Média Geral & $7,64 \mathrm{C}$ & $8,28 \mathrm{C}$ & $11,68 \mathrm{~B}$ & $14,11 \mathrm{~A}$ & - & - \\
\hline C.V. (\%) & 3,06 & 3,87 & 7,15 & 3,69 & - & - \\
\hline
\end{tabular}

O conteúdo de ácido ascórbico diferiu entre os acessos e entre os estádios de maturação (Tabela 9). No geral o conteúdo de ácido ascórbico aumentou nos estádios iniciais entre TV e AE, em seguida estabilizando até o ultimo estádio de maturação (PA).De acordo com Sacramento e Souza (2000), as frutas são fontes de calorias, sais minerais, vitaminas, fibras e compostos bioativos. Estes autores também relatam que o conteúdo destes compostos químicos varia de acordo com a espécie, e também depende de fatores genéticos, local de ocorrência, época de colheita, estádio de maturação, tratos culturais, entre outros.

Os acessos diferiram quanto o conteúdo de ácido ascórbico para todos os estádios de maturação avaliados. Para o estádio TV, o maior conteúdo de ácido ascórbico foi de frutos

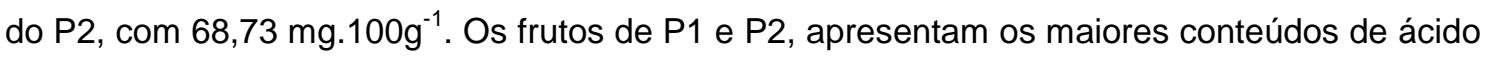
ascórbico nos estádios IP, AE e PA, com 60,53 e $63,14,54,26$ e 58,84 e 56,28 e 59,15 mg. $100 \mathrm{~g}^{-1}$, respectivamente (Tabela 9).

Houve diferença significativa entre os estádios de maturaçãopara os acessos P3, P4 e P5 (Tabela 4). Os frutos do acesso P3 apresentaram um aumento gradativo ao longo da maturação partido de $30,40 \mathrm{mg}^{1} 100 \mathrm{~g}^{-1}$ no estádio TV, para $36,94 \mathrm{mg} \cdot 100 \mathrm{~g}^{-1}$ no PA (Tabela9).

Para os frutos do acesso P4 observou-se um aumento do estádio TV $(36,31 \mathrm{mg} .100 \mathrm{~g}$ $\left.{ }^{1}\right)$ para IP $\left(44,02 \mathrm{mg} \cdot 100 \mathrm{~g}^{-1}\right)$, mantendo-se estável até o ultimo estádio avaliado PA $(46,91$ mg. $\left.100 \mathrm{~g}^{-1}\right)$. Já os frutos do acesso P5, apresentaram um aumento do estádio TV $(31,65$ $\left.\mathrm{mg} \cdot 100 \mathrm{~g}^{-1}\right)$ para IP $\left(46,24 \mathrm{mg} 100 \mathrm{~g}^{-1}\right)$, com posterior estabilidade até o ultimo estádio avaliado PA $\left(47,71 \mathrm{mg}^{1} 100 \mathrm{~g}^{-1}\right)$, (Tabela 9$)$.

Um aspecto importante sob a qualidade dos frutos desta espécie se dá principalmente devido ao elevado conteúdo de ácido ascórbico, pois os valores médios obtidos neste trabalho variaram de 45,46 a $49,40 \mathrm{mg} 100 \mathrm{~g}^{-1}$. O conteúdo de ácido ascórbico reportado neste trabalho, foi superior ao citado por Silva et al. (2003) com $38,86 \mathrm{mg} \cdot 100 \mathrm{~g}^{-1}$. Entretanto, 
Kohatsuet al. (2011) reportaram conteúdo de ácido ascórbico, variando de 35,9 a 54,2 $\mathrm{mg} .100 \mathrm{~g}^{-1}$ no armazenamento por doze dias a $8^{\circ} \mathrm{C}$, próximos aos deste trabalho.

\section{TABELA 9 - CONTEÚDO DE ÁCIDO ASCÓRBICO (mg.100g-1) EM FRUTOS DE DIFERENTES ACESSOS DA CAJÁ-MANGUEIRA EM DIFERENTES ESTÁDIOS DE MATURAÇÃO}

\begin{tabular}{|c|c|c|c|c|c|c|}
\hline \multirow{2}{*}{ Acessos } & \multicolumn{4}{|c|}{ Estádios de Maturacão } & \multirow{2}{*}{$\begin{array}{l}\text { Média } \\
\text { Geral }\end{array}$} & \multirow{2}{*}{ C.V. $(\%)$} \\
\hline & TV & IP & $\mathrm{AE}$ & PA & & \\
\hline $\mathrm{P} 1$ & $60,23 \mathrm{Ab}$ & $60,53 \mathrm{Aa}$ & $54,26 \mathrm{Aab}$ & $56,28 \mathrm{Aa}$ & 57,83 & 5,63 \\
\hline $\mathrm{P} 2$ & $68,73 \mathrm{Aa}$ & $63,14 \mathrm{Aa}$ & $58,84 \mathrm{Aa}$ & $59,15 \mathrm{Aa}$ & 62,47 & 6,12 \\
\hline P3 & $30,40 \mathrm{Bc}$ & $34,79 A C$ & $34,80 \mathrm{Ad}$ & $36,94 A c$ & 34,23 & 3,61 \\
\hline P4 & $36,31 \mathrm{Bc}$ & $44,02 \mathrm{Ab}$ & $45,95 \mathrm{Abc}$ & $46,91 \mathrm{Ab}$ & 43,30 & 3,98 \\
\hline P5 & $31,65 \mathrm{Bc}$ & $46,24 \mathrm{Ab}$ & $45,63 A c$ & $47,71 \mathrm{Ab}$ & 42,81 & 4,56 \\
\hline Média Geral & 45,46 & 49,75 & 47,90 & 49,40 & - & - \\
\hline C.V. (\%) & 5,08 & 5,71 & 6,53 & 3,87 & & - \\
\hline
\end{tabular}

* Médias seguidas de letras iguais, maiúsculas na linha e minúsculas na coluna, não diferem entre si pelo teste de Tukey, ao nível de $5 \%$ de probabilidade.

O conteúdo de ácido ascórbico do cajá-manga é superior ao de outras Spondias, por exemplo, para umbu-cajá (Spondiasp.) Santos et al. (2010), reportaram valores médios de 8,0 mg. $100 \mathrm{~g}^{-1}$. Silva et al. (2011), reportaram também para umbu-cajá (Spondia sp.) colhidos no estádio de maturação amarelo-alaranjado conteúdo de ácido ascórbico variando de 16,77 a $34,18 \mathrm{mg} \cdot 100 \mathrm{~g}^{-1}$.

O conteúdo de ácido ascórbico está presente naturalmente nas frutas é um parâmetro nutricional muito importante, o ácido ascórbico é definido como um composto antioxidante presente nos vegetais que minimiza a oxidação nas células causada pela geração de radicais livres em excesso, prevenindo doenças (Sharmaet al., 2008; Santos et al., 2010).

\section{CONCLUSÕES}

Os frutos dos diferentes acessos de cajá-mangueira apresentam um aumento no diâmetro e comprimento, na massa fresca, e um aumento na luminosidade e na intensidade da coloração da casca, em paralelo a transição da cor verde para uma cor em tons de amarelo intenso.

De modo geral os frutos da cajá-mangueira apresentam rendimento de polpa superior a $70 \%$, sendo esta porcentagem superior ao mínimo aceito para industrialização, atribuindo a estes frutos aptidão para consumo fresco.

Os frutos do acesso P1, P2 e P5 destacam-se com dupla finalidade, tanto para o consumo fresco como para a industrialização, por apresentarem rendimento de polpa, teor de SS superior e AT, superior ao mínimo aceitávelpelo padrão de identidade e qualidade (PIQ). Além de elevado conteúdo de ácido ascórbico, destacando-se os acessos P1 e P2 formam superiores aos demais por terem apresentado maior conteúdo de ácido ascórbico.

\section{AGRADECIMENTOS}

Ao Conselho Nacional de Desenvolvimento Científico e Tecnológico (CNPq) pelo auxílio financeiro e pela concessão da bolsa de iniciação cientifica ao primeiro autor. 


\section{ABSTRACT \\ QUALITY OF FRUIT OF CAJA-HOSPIT ACCESSES DURING MATURATION}

The objective of this study was to evaluate the changes in physical and physicochemical characteristics during the maturation of fruits of cajá-mangueira genotypes (Spondiasdulcis Parkinson). The fruits were obtained from five cajá-hose plants, of spontaneous occurrence, in five maturation stages (TV: totally green, IP: yellow pigmentation onset, AE: yellowish green and PA: predominantly yellow), and subsequently selected for uniformity of maturation. The experimental design was completely randomized, in a $5 \times 4$ two - factorial scheme, five genotypes and four maturation stages. The fruits were evaluated for Titratable Acidity (AT), soluble solids content (SS) and SS/AT ratio. It was verified that with the maturation advance the fruits of all the genotypes present an increase in the diameter and length, in the fresh mass, and an increase in the luminosity and the intensity of the color of the peel, in parallel the transition from green color to a color in shades of intense yellow. In general, the fruits of the cajá-hose have pulp yield higher than $70 \%$, being this percentage higher than the minimum accepted for industrialization. The fruits of the P1, P2 and P5 genotypes stand out as dual purpose, both for fresh consumption and for industrialization, as they present pulp yield, higher SS content and AT, higher than the minimum acceptable for the identity and quality standard (PIQ). In addition to high ascorbic acid content, the P1 and P2 genotypes are superior to the others because they have a higher content of ascorbic acid.

KEY-WORDS: SPONDIASDULCIS PARKINSON; EXOTIC FRUITFUL; ANALISIS PHYSICAL; ANALYSIS PHYSICO-CHEMICAL

\section{REFERÊNCIAS}

1 AROUCHA, E.M.M.; SOUZA, C.S.M.; SOUZA, A.E.D.; FERREIRA, R.M.A. AROUCHA FILHO, J.C. Qualidade pós-colheita da cajarana em diferentes estádios de maturação durante armazenamento refrigerado. Revista Brasileira de Fruticultura, v. 34, n. 2, p. 391-399, 2012.http://dx.doi.org/10.1590/S0100-29452012000200011

2 ASSOCIATION OF OFFICIAL ANALYTICAL CHEMISTRY. Official methods of analisys of the association of official analytical chemistry, Washington. 2005. $1015 \mathrm{p}$.

3 CAMPOS, C.O. Frutos de umbuzeiro (Spondias tuberosa arruda): características físicoquímicas durante seu desenvolvimento e na pós-colheita. 2007. 129 f. Tese (Doutorado em Agronomia / Horticultura) - Faculdade de Ciências Agronômicas, Universidade Estadual Paulista, Botucatu, 2007.

4 ChItARRA, M.I.F.; CHITARRA, A.B. Pós-colheita de Frutos e Hortaliças: Fisiologia e manuseio.2d. Lavras: UFLA/FAEPE, 2009. 523 p.

5 COSTA, F.R.; NEDER, D.G.; REGO E.R.; REGO, M.M.; NEDER, D.G.; SILVA, S.M.; SCHUNEMANN, A.P.P.Análise biométrica de frutos de umbuzeiro do semiárido brasileiro. BioscienceJournal, v. 31, n. 3, p. 682-690, 2015.

6 DAMIANI, C.; SILVA, F.A.; AMORIM, C.C.M.; SILVA, S.T.P.; BASTOS, I.M.; ASQUIERI, E.R.; VERA, R. Néctar misto de cajá-manga com hortelã: caracterização química, microbiológica e sensorial. Revista Brasileira de Produtos Agroindustriais, v. 13, n. 3, p. 299-307, 2011.http://dx.doi.org/10.15871/1517-8595/rbpa.v13n3p301-309 
DANTAS JUNIOR, O.R.D. Qualidade e capacidade antioxidante total de frutos de acessos de umbuzeiro oriundos do semi-árido nordestino. 2008. 106 f. Tese (Doutorado em Agronomia) Universidade Federal da Paraíba, Areia-PB, 2008.

8 DANTAS, A.L.; SILVA, S.M.; DANTAS, R.L. SOUSA, A.S.B.; SCHUNEMANN, A.P.P. Desenvolvimento, fisiologia da maturação e indicadores do ponto de colheita de frutos da umbugueleira (Spondiassp.). Revista Brasileira de Fruticultura, v. 38, n. 1, p. 033-042, 2016. http://dx.doi.org/10.1590/0100-2945-271/14

9 FERREIRA, D.F. Sisvar: A Guide for its Bootstrap procedures in multiple comparisons. Ciência \&Agrotecnologia, v.38, n.2, p.109-112, 2014.http://dx.doi.org/10.1590/S1413-70542014000200001

10 FRANQUIN, S.; MARCELIN, O.; AURORE, G.; REYNES, M.; BRILLQUET, J.M. Physicochemical characterisation of the mature-green Golden apple (SpondiascythereaSonnerat). Fruits, v.60, n. 3, p.203-210, 2005.http://dx.doi.org/10.1051/fruits:2005027

11 FRANQUIN, S.; MARCELIN, O.; REYNES, M.; BRILLOUET, J.M. Characterization of starch and cell walls from mature-green ambarella (SpondiascythereaSonnerat) and their enzymatic hydrolysis. European Food Research and Technology, v. 226, n. 4, p. 905-913, 2008.http://dx.doi.org/10.1007/s00217-007-0613-0

GONDIM, P.J.S.; SILVA, S.M.; PEREIRA, W.E.; DANTAS, A.L.; CHAVES NETO, J.R.; SANTOS, L.F. Qualidade de frutos de acessos de umbu-cajazeira (Spondiassp.). Revista Brasileira de Engenharia Agrícola e Ambiental, v. 17, n. 11, p. 1217-1221, 2013.http://dx.doi.org/10.1590/S1415-43662013001100013

13 GRAHAM, O.S.; WICKHAM, L.D.; MOHAMMED, M. Growth, development and quality attributes of miniature golden apple fruit (SpondiascythereaSonn). Journal of Food, Agriculture \& Environment, v. 2, n. 1, p. 90-94, 2004.

14 KLUGE, R.A.; NACHTIGAL, J.C.; FACHINELLO, J.C. et al. Fisiologia e manejo pós-colheita de frutas de clima temperado.2d. Pelotas: UFPEL, 2002. $163 \mathrm{p}$.

15 KOHATSU, D.S.; ZUCARELI, V.; BRAMBILLA, W.P.; EVANGELISTA, R.M. Qualidade de frutos de cajá-manga armazenados sob diferentes temperaturas. Revista Brasileira de Fruticultura, v. (E), p. 344-349, 2011.http://dx.doi.org/10.1590/S0100-29452011000500043

16 LAGO-VANZELA, E.S.; RAMIN, P.; UMSZA-GUEZ, M.A.; SANTOS, G.V.; GOMES, E; SILVA, R. Chemicalandsensorycharacteristicsofpulpandpeel 'cajá-manga' (SpondiascythereaSonn.) jelly. Ciência e Tecnologia de Alimentos, v. 31, n. 2, p. 398-405, 2011. http://dx.doi.org/10.1590/S010120612011000200018

17 LIMA, E.D.P.A.; LIMA, C.A.A.; ALDRIGUE, M.L.; GONDIM, P.J.S. Caracterização física e química dos frutos da umbu-cajazeira (Spondiasspp.) em cinco estádios de maturação, da polpa congelada e néctar. Revista Brasileira de Fruticultura, v. 24, n. 2, p. 338-343, 2002.http://dx.doi.org/10.1590/S0100-29452002000200013

18 LIRA JÚNIOR, J.S.; BEZERRA, J.E.F.; MOURA R.J.M. SANTOS, V.F. Repetibilidade da produção, número e peso de fruto em cirigueleira (SpondiaspurpureaL.). Revista Brasileira de Fruticultura, v. 36, n. 1, p. 214-220, 2014. http://dx.doi.org/10.1590/0100-2945-294/13

19 LOPES, M.F. Fisiologia da maturação e conservação pós-colheita do acesso umbu-laranja (Spondias tuberosa Arruda Câmara). 2007. 144 f. Dissertação (Mestrado em Ciência e Tecnologia de Alimentos) - Universidade Federal da Paraíba, João Pessoa, 2007.

20 LORENZI, H.; BACHER, L.; LACERDA, M.; SARTORI, S. Frutas brasileiras e exóticas cultivadas: de consumo in natura. 1. ed. São Paulo: INSTITUTO PLANTARUM, 2002. 672 p.

21 MARTINS, L.P.; SILVA, S.M.; ALVES, R.E.; FILGUEIRAS, H.A.C. Desenvolvimento de frutos de cirigueleira (SpondiaspurpureaL.). Revista Brasileira de Fruticultura, v. 25, n. 1, p. 11-14, 2003.http://dx.doi.org/10.1590/S0100-29452003000100005

22 MCATEE, P.; KARIM, S.; SCHAFFER, R.; DAVID, K. A dynamic interplay between phytohormones is required for fruit development, maturation, and ripening. Frontiers in Plant Science, v. 4, n. 79, p. 17, 2013.http://dx.doi.org/10.3389/fpls.2013.00079 
MOURA, F.T.; SILVA, S.M.; MARTINS, L.P.; MENDONÇA, R.M.N.; ALVES, R.E.; FILGUEIRAS, L.A.C.Evolução do Crescimento e da Maturação de Frutos de Cajazeira (SpondiasmombinL). Proceedings of Thelnteramerican Society for Tropical Horticulture, v. 47, p. 231-233, 2003.

MOURA, F.T.; SILVA, S.M.; SCHUNEMANN, A.P.P.; MARTINS, L.P. Frutos do umbuzeiro armazenados em diferentes estádios de maturação. Revista Ciência Agronômica, v. 44, n. 4, p. 764-772, 2013. http://dx.doi.org/10.1590/S1806-66902013000400013

PINTO W.S.; DANTAS, A.C.V.L.; FONSECA, A.A.O.; LEDO, C.A.S.; JESUS, S.C.; CALAFANGE, P.L.P.; ANDRADE, E.M. Caracterização física, físico-químicae química de frutos de acessos de cajazeiras. Pesquisa AgropecuáriaBrasileira, v. 38, n. 9, p. 1059-1066, 2003.http://dx.doi.org/10.1590/S0100-204X2003000900006

SACRAMENTO, C.K.; MATOS, C.B.; SOUZA, C.N.; BARRETO, W.S.; FARIA, J.C. Características físicas, físico-químicas e químicas de cajás oriundos de diversos municípios da região sul da Bahia.Magistra, v. 19, n. 4, p. 283-289, 2007.

SANTOS, M.B.; CARDOSO, R.L.; FONSECA, A.A.O.; CONCEIÇÃO, M.N. Caracterização e qualidade de frutos de umbu-cajá (Spondias tuberosa $x$ S. mombin) provenientes Do Recôncavo sul da Bahia.Revista Brasileira de Fruticultura, v. 32, n. 4, p. 1089-1097, 2010. http://dx.doi.org/10.1590/S0100-29452011005000015

SHARMA, M.; SITBON, C.; SUBRAMANIAN, J.; PALIYATHIN, G. Changes in nutritional quality of fruits and vegetables during storage. In: PALIYATH, G.; MURR, D. P.; HANDA, A. K.; LURIE, S. (eds) Postharvest Biology and Technology of Fruits, Vegetables and Flowers. WileyBlackwellPublishing. 443-466. 2008.

30 SILVA, D.B.; SILVA, J.A.; JUNQUEIRA, N.T.V.; ANDRADE, L.R.M. Frutas do cerrado. Brasília: Embrapa Informação Tecnológica/UFC, 2003. 179p.

31 SILVA, F.V.G. Maturação, compostos bioativos e capacidade antioxidante de frutos de acessos de cajazeira do BAG Emepa-PB. 2010. 191 f. Tese (Doutorado em Ciência e Tecnologia de Alimentos) - Universidade Federal da Paraíba, João Pessoa, 2010.

SILVA, F.V.G.; SILVA, S.M.; SILVA, G.C.; MENDONÇA, R.M.N.; ALVES, R.E.; DANTAS, A.L. Bioactive compounds and antioxidant activity in fruits of clone and ungrafted genotypes of yellow mombin tree. Ciência Tecnologia de Alimentos, v. 32, n. 4, p. 685-691, 2012. http://dx.doi.org/10.1590/S0101-20612012005000101

33 SILVA, F.V.G.; SILVA, S.M.; SILVA, G.C.; SCHUNEMANN, A.P.P. Quality and antioxidant activity during ripening of fruits from yellow mombin (Spondiasmombin L.) genotypes. Actahorticulturae, $\mathrm{n}$. 1012, p. 843-848, 2013.http://dx.doi.org/10.17660/ActaHortic.2013.1012.114

SILVA, G.G.; MORAIS, P.L.D.; ROCHA, R.H.C.; SANTOS, E.C.; SARMENTO, J.D.A. Caracterização do fruto de cajaranazeira em diferentes estádios de maturação. Revista Brasileira de Produtos Agroindustriais, v. 1, n. 2, p. 159-163, 2009.http://dx.doi.org/10.15871/1517-8595/rbpa.v11n2p159163

SILVA, L.R.; ALVES, R.E.; ARAGÃO, F.A.Z.; SILVA, S.M.; MAIA, L.K.; NOGUEIRA, D.H. Qualidade de frutos de acessos de umbu-cajazeiras (Spondiassp.) oriundos da microrregião de Iguatu-CE. Scientia Plena, v. 7, n. 8, p. 1-7, 2011.http://dx.doi.org/10.3895/rbta.v9n1.1699 MONTALVO, 1967, 428p.

WILLS, R.B.H.; WIDJANARKO, S.B. Changes in physiology, composition and sensory characteristics of Australian papaya during ripening. Australian Journal of Experimental Agriculture, v. 35, p. 11273-1176, 1995.http://dx.doi.org/10.1071/EA9951173 